\title{
Decreased insulin secretion and increased risk of type 2 diabetes associated with allelic variations of the WFS1 gene: the Data from Epidemiological Study on the Insulin Resistance Syndrome (DESIR) prospective study
}

\author{
N. Cheurfa • G. M. Brenner • A. F. Reis • D. Dubois-Laforgue $・$ R. Roussel • J. Tichet • \\ O. Lantieri • B. Balkau • F. Fumeron • J. Timsit • M. Marre • G. Velho
}

Received: 2 August 2010 /Accepted: 22 October 2010 /Published online: 3 December 2010

(C) Springer-Verlag 2010

\begin{abstract}
Aims/hypothesis We investigated associations of allelic variations in the WFS1 gene with insulin secretion and risk of type 2 diabetes in a general population prospective study. Methods We studied 5,110 unrelated French men and women who participated in the prospective Data from Epidemiological Study on the Insulin Resistance Syndrome
\end{abstract}

Electronic supplementary material The online version of this article (doi:10.1007/s00125-010-1989-0) contains a list of members of the DESIR Study Group, and is available to authorised users.

\footnotetext{
N. Cheurfa $\cdot$ G. M. Brenner ' R. Roussel · F. Fumeron •

M. Marre $\cdot$ G. Velho $(\square)$

INSERM, Research Unit 695,

16 rue Henri Huchard,

75018 Paris, France

e-mail: gilberto.velho@inserm.fr

G. M. Brenner

Post-Graduation Program in Health Sciences,

Federal University of Health Sciences of Porto Alegre,

Porto Alegre, Brazil
}

\section{A. F. Reis}

Laboratory of Molecular Endocrinology,

Federal University of São Paulo,

São Paulo, Brazil

D. Dubois-Laforgue $\cdot$ J. Timsit

Department of Immunology and Diabetology,

Assistance Publique Hôpitaux de Paris - Cochin Hospital,

Paris, France

D. Dubois-Laforgue $\cdot$ J. Timsit

UFR de Médecine, Université Paris Descartes (Paris 5),

Paris, France
(DESIR) study. Additional cross-sectional analyses were performed on 4,472 French individuals with type 2 diabetes and 3,065 controls. Three single nucleotide polymorphisms (SNPs) were genotyped: rs10010131, rs1801213/rs7672995 and rs734312.

Results We observed statistically significant associations between the major alleles of the three variants and prevalent

R. Roussel $\cdot$ F. Fumeron $\cdot$ M. Marre

UFR de Médecine, Université Paris Diderot (Paris 7),

Paris, France

R. Roussel $\cdot$ M. Marre

Department of Diabetology, Endocrinology and Nutrition, Assistance Publique Hôpitaux de Paris - Bichat Hospital, Paris, France

J. Tichet $\cdot$ O. Lantieri Institut Inter Régional pour la Santé (IRSA), La Riche, France

B. Balkau

INSERM, U1018, CESP Centre for research in Epidemiology and Population Health,

U1018 Villejuif, France

B. Balkau

UMRS 1018, Université Paris Sud (Paris 11),

Villejuif, France 
type 2 diabetes in the DESIR cohort at baseline. Cox analyses showed an association between the G-allele of rs10010131 and incident type 2 diabetes (HR 1.34, 95\% CI $1.08-1.70, p=0.007)$. Similar results were observed for the G-allele of rs1801213 and the A-allele of rs 734312 . The GGA haplotype was associated with an increased risk of diabetes as compared with the ACG haplotype (HR 1.26, $95 \%$ CI $1.04-1.42, p=0.02$ ). We also observed statistically significant associations of the three SNPs with plasma glucose, $\mathrm{HbA}_{1 \mathrm{c}}$ levels and insulin secretion at baseline and throughout the study in individuals with type 2 diabetes or at risk of developing diabetes. However, no association was observed in those who remained normoglycaemic at the end of the follow-up. Associations between the three variants and type 2 diabetes were replicated in cross-sectional studies of type 2 diabetic patients in comparison with a non-diabetic control group.

Conclusions/interpretation The most frequent haplotype at the haplotype block containing the WFS1 gene modulated insulin secretion and was associated with an increased risk of type 2 diabetes.

Keywords Endoplasmic reticulum stress · Genetic epidemiology Population studies $\cdot$ Wolframin

$\begin{array}{ll}\begin{array}{l}\text { Abbreviations } \\ \text { ATF6 } \alpha\end{array} & \begin{array}{l}\text { Activating transcription factor } 6 \alpha \\ \text { Data from Epidemiological Study on the } \\ \text { Insulin Resistance Syndrome }\end{array} \\ \text { DIABHYCAR } & \begin{array}{l}\text { The Diabetes, Hypertension, } \\ \text { Cardiovascular Events, and Ramipril study }\end{array} \\ \text { ER } & \begin{array}{l}\text { Endoplasmic reticulum } \\ \text { Fasting plasma glucose }\end{array} \\ \text { FPG } & \text { Glucagon-like peptide 1 } \\ \text { GLP-1 } & \text { Impaired fasting glucose } \\ \text { IFG } & \text { HOMA index of beta cell function } \\ \text { HOMA\%B } & \text { HOMA index of insulin sensitivity } \\ \text { HOMA\%S } & \text { Necker and Cochin Hospitals } \\ \text { NCH } & \text { Normal fasting glucose } \\ \text { NFG } & \text { Single nucleotide polymorphism } \\ \text { SNP } & \text { Wolframin } \\ \text { WFS1 } & \end{array}$

\section{Introduction}

Wolframin (WFS1) is a transmembrane protein of the endoplasmic reticulum (ER), found ubiquitously but with higher levels in pancreatic beta cells and specific neurons of the central nervous system [1]. WFS1 is a component of the unfolded protein response following ER stress [2]. It controls a negative regulatory feedback loop of the ER stress signalling network, acting as an ER stress signalling suppressor [3]. ER stress signalling is tightly regulated in pancreatic beta cells to produce appropriate amounts of insulin in response to the frequent fluctuations of blood glucose levels $[4,5]$. WFS1 is upregulated during insulin secretion and plays an important role in maintaining ER homeostasis and cell integrity in pancreatic beta cells [2]. Inactivation of the wolframin gene $\left(W f_{S} l\right)$ in beta cells of rodents causes ER stress and beta cell death by accelerated apoptosis $[2,3,6]$.

Mutations in WFS1 are responsible for Wolfram syndrome $[7,8]$, a rare recessive disorder characterised by diabetes mellitus, optic atrophy and other neurological and endocrine abnormalities [9]. An increased prevalence of diabetes mellitus was reported in first-degree relatives of patients with Wolfram syndrome [10], suggesting a possible effect of WFS1 mutation heterozygosis. More recently, common variants of WFS1 were shown to be associated with type 2 diabetes in populations of European descent [11-13]. Associations of these variants with insulin secretion in response to oral or intravenous glucose was observed in some [14, 15], but not all studies [16-18]. In the present study, we investigated associations of allelic variations in the WFS1 gene with insulin secretion, insulin sensitivity and the risk of hyperglycaemia (impaired fasting glucose [IFG]) and of type 2 diabetes in a 9 year population-based prospective cohort. We tested the hypothesis that discrepancies in the associations between WFS1 variants and insulin secretion in previous reports could be related to differences in glycaemic and/or diabetes risk status of studied populations. For the purpose of replication, associations between WFS1 variants and diabetes were also investigated in three independent type 2 diabetes crosssectional cohorts.

\section{Methods}

Participants in prospective study: the DESIR cohort The Data from Epidemiological Study on the Insulin Resistance syndrome (DESIR) study was a 9 year prospective study conducted in 5,212 men and women from the French general population $[19,20]$. Participants were recruited in ten healthcare centres in the western central part of France on the occasion of periodic health check-ups offered by Social Security. The study protocol included clinical and biological evaluations at inclusion and at visits after 3, 6 and 9 years of follow-up. Glycaemic status was classified in three groups, defined on the basis of fasting plasma glucose (FPG) and hypoglycaemic medication. Normal fasting glucose (NFG) was defined as FPG $<6.1 \mathrm{mmol} / 1$ and IFG as FPG between 6.1 and $6.99 \mathrm{mmol} / \mathrm{l}$, in the absence of treatment with a hypoglycaemic agent for both classes. Type 2 diabetes was defined as $\mathrm{FPG} \geq 7.0 \mathrm{mmol} / \mathrm{l}$ or as 
FPG $<7.0 \mathrm{mmol} / \mathrm{l}$ in the presence of treatment with a diabetes drug and a previous diagnosis of diabetes. The research protocol was approved by the ethics committee of Bicêtre Hospital and all participants signed an informed consent form. At baseline, 4,634 individuals (88.9\%) had NFG, $342(6.6 \%)$ IFG and $134(2.6 \%)$ diabetes. The glycaemic status of 102 participants $(1.9 \%)$ could not be ascertained at baseline and they were excluded from the analyses. NFG individuals lost during follow-up and whose final glycaemic status could not be determined $(n=1,003)$ were excluded from the analyses of incidence. For the purpose of this investigation, we have considered the incidence of type 2 diabetes and the incidence of hyperglycaemic events (NFG to IFG, NFG to diabetes or IFG to diabetes change) during follow-up. The incidence of type 2 diabetes was $5.1 \%$ (203 cases out of 3,973 NFG or IFG cases at baseline), while the incidence of hyperglycaemic events was $16.7 \%$ (663 cases out of 3,973 NFG or IFG cases at baseline).

Participants in cross-sectional studies: DIABHYCAR, DIABHYCAR Gene and NCH cohorts The Diabetes, Hypertension, Cardiovascular Events, and Ramipril (DIABHYCAR) study was a 6 year double-blind multicentre multinational clinical trial conducted in 4,912 men and women who had type 2 diabetes, were aged 50 years or older at baseline and were selected on the basis of persistent micro- or macroalbuminuria without renal failure. The trial tested in a parallel design and vs placebo whether a low dose of ramipril that was able to reduce urinary albumin concentration would also reduce cardiovascular and/or renal events such as myocardial infarction, stroke, acute heart failure, end-stage renal failure and cardiovascular death. The study design, methods and results of the trial (which were negative regarding the drug effect) have been published previously $[21,22]$. DNA banking was undertaken for the 3,137 participants recruited in France. The DIABHYCAR Gene cohort was recruited concomitantly to DIABHYCAR. It included 607 men and women with type 2 diabetes, aged 50 years or older at baseline and not presenting with microor macroalbuminuria. The study protocols of the two cohorts were approved by the Angers University Hospital Ethics Committee. The Necker and Cochin Hospitals (NCH) cohort included type 2 diabetic men and women consecutively recruited at the diabetes departments of $\mathrm{NCH}$ in Paris [23]. The present investigation included 728 individuals of European descent. Clinical and biological data were obtained from medical records. The study protocol was approved by the Ethics Committee of Hôpital Necker. For the purpose of these cross-sectional studies, the three cohorts of type 2 diabetic patients were compared with a control group of 3,065 participants from the DESIR cohort who had remained normoglycaemic at the 9-year follow-up (DESIR controls). This group from the French general population has been extensively used as a control group in genetic studies [24, 25].

DNA studies The WFS1 gene spans $\sim 33.4 \mathrm{kbp}$ on chromosome 4 p16 and is contained in a single haplotype block. Three single nucleotide polymorphisms (SNPs) were genotyped using assay by design kits from Applied Biosystems (Life Technologies, Carlsbad, CA, USA): rs10010131 (G>A, intron 4), rs1801213/rs7672995 (G>C, R228R, exon 6) and rs734312 (A $>\mathrm{G}, \mathrm{H} 611 \mathrm{R}$, exon 8). The SNPs were chosen in HapMap on the basis of capturing information on $\sim 80 \%$ of the allelic variation of SNPs with minor allele frequency $\geq 5 \%$ at $r^{2}>0.8$ in the haplotype block containing WFS1.

Computations and statistical analyses HOMA index of beta cell function ( $\mathrm{HOMA} \% \mathrm{~B})$ and of insulin sensitivity (HOMA\%S) [26], based on fasting levels of glucose and insulin, were computed using the Microsoft Excel spreadsheet implementation of the HOMA Calculator (v2.2) available at www.dtu.ox.ac.uk/Homacalculator/download. php (accessed 1 March 2006). Before genotype-related statistical analyses were performed, genotypes were verified to be in Hardy-Weinberg equilibrium in all groups of participants. Genotype associations with diabetes or hyperglycaemia were assessed by regression models. Cox proportional hazards survival regression analyses were used to examine the effect of explanatory variables on timerelated survival (disease-free) rates in prospective analyses. Kaplan-Meier curves were used to plot survival (diseasefree) rates over time according to genotype. Logistic regression analyses were used for cross-sectional analyses. Hazard ratios or odds ratios, respectively, with 95\% CIs, were computed in these analyses for the risk allele. Adjustments for clinical and biological variables were carried out by including these variables as covariates in the regression model. The study had power $>0.99$ to detect associations of the SNPs with the incidence of diabetes in the DESIR study (for $\mathrm{HR} \geq 1.2$ and $\alpha=0.05$ ) and with the prevalence of diabetes in the cross-sectional study (for OR $\geq 1.3$ and $\alpha=$ 0.05 ). It had power $>0.80$ (for $\alpha=0.05$ ) to detect differences in quantitative traits $>10 \%$ between groups.

Comparisons of anthropometric, clinical and biological phenotypes between groups were assessed by ANOVA, analyses of covariance, contingency table $\chi^{2}$ test and Fisher's exact test. For the comparisons of FPG, $\mathrm{HbA}_{1 \mathrm{c}}$, insulin, $\mathrm{HOMA} \% \mathrm{~B}$ and $\mathrm{HOMA} \% \mathrm{~S}$ between genotypes, interaction between the glycaemic status at follow-up (NFG or IFG/diabetes) and genotype was assessed by including in the regression model a 'crossed' compound covariable (glycaemic status/genotype). Stratification by glycaemic status of genotype-related effects on phenotypic traits was 
then performed by nesting the genotype variable within the glycaemic status variable in the regression analysis. This results in the computation of separate statistical effects for NFG and IFG/diabetes subgroups of individuals, adjusted for multiple comparisons due to the stratification by glycaemic status. For all analyses, data were logtransformed for the analyses when the normality of the distribution was rejected by the Shapiro-Wilk W test. Results are expressed as means \pm SEM. $p<0.05$ was considered significant. Giving the strong linkage disequilibrium between the SNPs (see below), no $p$ value correction for multiple SNP testing was performed, as it was considered that they were not independent analyses. Statistics were performed with JMP software (SAS Institute, Cary, NC, USA). Power calculations were performed with Quanto v1.2.4 (http://hydra.usc.edu/gxe; accessed 10 June 2009) and Logistic_Cox_Power (http://hedwig.mgh. harvard.edu/biostatistics/node/19; accessed 10 June 2009). Determination of linkage disequilibrium between SNPs and between haplotype frequencies, and computation of hazard and odd ratios associated with haplotypes were performed with THESIAS software v3.1 (http://ecgene.net/genecanvas/ uploads/THESIAS3.1/Documentation3.1.htm; accessed 15 January 2010) [27].

\section{Results}

Prospective study: DESIR cohort Prevalence studies were conducted in 5,110 participants from the DESIR cohort (Table 1). The combined prevalence at baseline of IFG/ diabetes according to genotype was: $10.3 \%(\mathrm{GG}), 9.3 \%$ (GA) and 7.5\% (AA) for rs10010131; 9.8\% (GG), 9.3\% (GC) and 7.5\% (CC) for rs 1801213 ; and 10.4\% (AA), 9.4\% (AG) and 7.8\% (GG) for rs 734312. Logistic regression analyses showed associations of the major allele of the three variants with diabetes and with the combined prevalence of IFG/diabetes at baseline (Table 2). When we considered the prevalence of IFG only, associations were also observed for rs10010131 and rs734312, while only a trend towards an association was observed for rs1801213 (Table 2).

The main baseline characteristics of incident cases of type 2 diabetes or hyperglycaemic events are shown in Electronic supplementary material (ESM) Table 1. The incidence of type 2 diabetes according to genotype was: 5.5\% (GG), 5.4\% (GA) and 3.6\% (AA) for rs 10010131 ; $5.2 \%(\mathrm{GG}), 5.4 \%(\mathrm{GC})$ and $3.3 \%(\mathrm{CC})$ for rs 1801213 ; and $5.5 \%(\mathrm{AA}), 5.6 \%(\mathrm{AG})$ and $4.0 \%(\mathrm{GG})$ for $\mathrm{rs} 734312$. This suggests a dominant effect of the major allele for all SNPs (Fig. 1). Cox proportional hazards survival regression analyses showed a statistically significant association between the G-allele of rs10010131 and the incidence of type 2 diabetes (HR 1.34, 95\% CI 1.08-1.70, $p=0.007$ for a dominant GX vs AA model; Table 3). Similar results were observed for the G-allele of rs 1801213 (HR 1.44, 95\% CI $1.10-1.99, p=0.007$ ) and for the A-allele of rs 734312 (HR $1.22,95 \%$ CI $1.02-1.48, p=0.03$ ). Haplotype frequencies were determined for incident cases of diabetes and for participants who did not develop diabetes during the follow-up (NFG/IFG censored individuals) and Cox survival (disease-free) analyses were performed for the haplotypes. There was a strong linkage disequilibrium between the three SNPs $\left(0.74 \leq \mathrm{D}^{\prime} \leq 0.93\right)$, with haplotypes composed of the major (GGA) or minor (ACG) alleles of the variants representing $\sim 77 \%$ of all haplotypes. GGA frequency was $51.0 \%$ in incident cases of type 2 diabetes and $47.6 \%$ in NFG/IFG censored individuals, while ACG frequencies were $26.6 \%$ and $29.2 \%$, respectively. The GGA haplotype was significantly associated with an increased risk of diabetes compared with the ACG haplotype (HR
Table 1 Clinical and biological characteristics at baseline according to glycaemic status, the DESIR cohort

Except where otherwise indicated, data are expressed as mean \pm SEM

Comparisons are by ANOVA

\begin{tabular}{lllll}
\hline Characteristic & \multicolumn{2}{l}{ Glycaemic status } & & \\
\cline { 2 - 5 } & NFG & IFG & Type 2 diabetes & $p$ value \\
\hline$n$ & 4,634 & 342 & 134 & \\
Age (years) & $46.8 \pm 0.1$ & $51.9 \pm 0.5$ & $55.0 \pm 0.7$ & $<0.0001$ \\
Sex & & & & $<0.0001$ \\
$\quad$ Men (\%) & 47 & 73 & 71 & \\
$\quad$ Women (\%) & 53 & 27 & 29 & $<0.0001$ \\
BMI (kg/m ${ }^{2}$ ) & $24.5 \pm 0.1$ & $26.9 \pm 0.2$ & $28.9 \pm 0.3$ & $<0.0001$ \\
FPG (mmol/1) & $5.20 \pm 0.01$ & $6.40 \pm 0.03$ & $8.40 \pm 0.06$ & $<0.0001$ \\
HbA 1 (\%) & $5.40 \pm 0.01$ & $5.83 \pm 0.03$ & $6.87 \pm 0.04$ & $<0.0001$ \\
Fasting insulin (pmol/1) & $45 \pm 1$ & $65 \pm 2$ & $87 \pm 2$ & $<0.0001$ \\
HOMA\%B (\%) & $77 \pm 1$ & $65 \pm 1$ & $54 \pm 2$ & $<0.0001$ \\
HOMA\%S (\%) & $146 \pm 1$ & $100 \pm 3$ & $85 \pm 5$ & \\
\hline
\end{tabular}


Table 2 Genotype frequency according to glycaemic status at baseline, the DESIR cohort

\begin{tabular}{|c|c|c|c|c|c|c|c|c|c|}
\hline \multirow[t]{2}{*}{ Genotype by SNP } & \multicolumn{3}{|c|}{ Glycaemic status } & \multicolumn{6}{|c|}{ OR $(95 \% \mathrm{CI})$ for comparison of genotype frequency by glycaemic status } \\
\hline & $\mathrm{NFG}^{\mathrm{a}}$ & $\mathrm{IFG}^{\mathrm{b}}$ & $\mathrm{DM}^{\mathrm{c}}$ & IFG vs NFG & $p$ value & DM vs NFG & $p$ value & IFG/DM vs NFG & $p$ value \\
\hline \multicolumn{10}{|l|}{ rs10010131 } \\
\hline GG & 0.369 & 0.385 & 0.466 & $1.39(1.00-1.94)$ & 0.05 & $1.98(1.15-3.45)$ & 0.01 & $1.50(1.11-2.02)$ & 0.008 \\
\hline GA & 0.482 & 0.506 & 0.397 & & & & & & \\
\hline AA & 0.149 & 0.109 & 0.137 & & & & & & \\
\hline \multicolumn{10}{|l|}{ rs1801213 } \\
\hline GG & 0.472 & 0.485 & 0.530 & $1.38(0.97-1.98)$ & 0.07 & $1.47(1.02-2.11)$ & 0.04 & $1.43(1.05-1.97)$ & 0.02 \\
\hline $\mathrm{GC}$ & 0.433 & 0.452 & 0.366 & & & & & & \\
\hline $\mathrm{CC}$ & 0.095 & 0.063 & 0.104 & & & & & & \\
\hline \multicolumn{10}{|l|}{ rs734312 } \\
\hline AA & 0.292 & 0.317 & 0.354 & $1.47(1.06-2.04)$ & 0.02 & $1.62(1.0-2.72)$ & 0.05 & $1.51(1.13-2.01)$ & 0.005 \\
\hline $\mathrm{AG}$ & 0.494 & 0.515 & 0.446 & & & & & & \\
\hline GG & 0.214 & 0.168 & 0.200 & & & & & & \\
\hline
\end{tabular}

Values are ORs for the major alleles in a co-dominant model, adjusted for sex, and for age and BMI at baseline

${ }^{\mathrm{a}} n=4,634,{ }^{\mathrm{b}} n=342,{ }^{\mathrm{c}} n=134$

DM, type 2 diabetes mellitus

$1.26,95 \%$ CI 1.04-1.42, $p=0.02$, comparison adjusted for sex, age and BMI).

The incidence of hyperglycaemic events according to genotype was: $17.1 \%$ (GG), 16.5\% (GA) and 16.7\% (AA) for $\mathrm{rs} 10010131 ; 17.4 \%(\mathrm{GG}), 16.5 \%(\mathrm{GC})$ and $15.3 \%$ (CC) for $\mathrm{rs} 1801213$; and 16.2\% (AA), 17.7\% (AG) and 15.9\% (GG) for rs734312. Cox proportional hazards survival regression analyses showed a trend towards an association between the G-allele of rs1801213 and the incidence of hyperglycaemic events (HR 1.11, 95\% CI 0.99-1.25, $p=$ 0.08 for a co-dominant additive model) (ESM Table 2), while no association was observed for the other variants.

We assessed the impact of the SNPs on intermediate phenotypes such as $\mathrm{FPG}, \mathrm{HbA}_{1 \mathrm{c}}$, fasting insulin, and $\mathrm{HOMA} \% \mathrm{~B}$ (insulin secretion) and HOMA\%S (insulin sensitivity) indices. Analyses were performed with baseline values and with all available values collected throughout the study (baseline plus values at 3,6 and 9 year visits). Results at baseline for rs10010131 genotypes are shown in Table 4. These phenotypic analyses revealed a strong interaction between genotype and glycaemic status at the end of the study (NFG or IFG/diabetes). Genotype-related effects on intermediate traits were observed in the subset of the population presenting with IFG or diabetes at the end of the study, but not in individuals with NFG. In the former subset of the population, significantly higher FPG and $\mathrm{HbA}_{1 \mathrm{c}}$ levels and lower HOMA\%B levels were observed in carriers of the GG risk genotype than in AA carriers. HOMA $\%$ S was not significantly different between groups. The exclusion of participants receiving sulfonylurea $(n=15)$, metformin $(n=15)$ or both $(n=12)$ had no significant effect on the computations. Comparable results were observed when we considered the values of these variables collected throughout the study (ESM Table 3). Similar results were
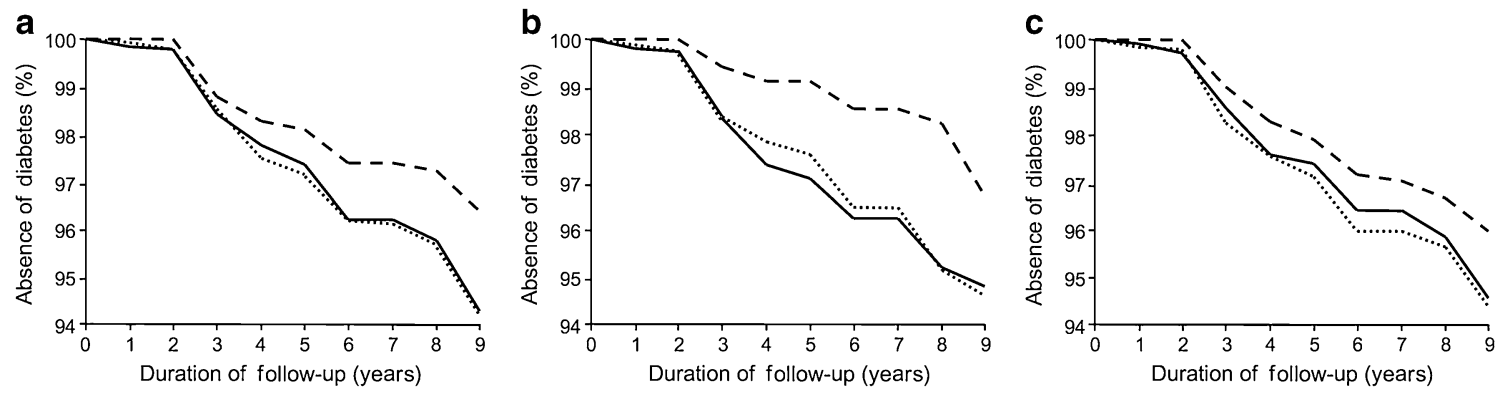

Fig. 1 Kaplan-Meier survival (disease-free) curves for the DESIR cohort during follow-up according to genotype. a rs10010131, AA (dashed line), GA (dotted line), GG (solid line); (b) rs1801213, CC (dashed line), GC (dotted line), GG (solid line); and (c) rs734312, GG (dashed line), AG (dotted line), AA (solid line) 
Table 3 Genotype frequency according to incidence of diabetes during follow-up, the DESIR cohort

Hazard ratios (HR) for the major alleles in a dominant model $(M X$ vs $\mathrm{mm}$ ) determined by Cox proportional hazards survival regression analyses adjusted for sex, and for age and BMI at baseline. Time to event was defined as the number of years of follow-up until the diagnosis of type 2 diabetes or the duration of follow-up for rightcensored participants without diabetes at the end of follow-up ${ }^{\mathrm{a}} n=3770,{ }^{\mathrm{b}} n=203$

\begin{tabular}{|c|c|c|c|c|}
\hline \multirow[t]{2}{*}{ Genotype by SNP } & \multicolumn{2}{|c|}{ Incidence of diabetes } & \multirow[t]{2}{*}{$\mathrm{HR}(95 \% \mathrm{CI})$ for incidence } & \multirow[t]{2}{*}{$p$ value } \\
\hline & $\mathrm{No}^{\mathrm{a}}$ & Yes $^{\mathrm{b}}$ & & \\
\hline \multicolumn{5}{|l|}{ rs10010131 } \\
\hline GG & 0.372 & 0.395 & $1.34(1.08-1.70)$ & 0.007 \\
\hline GA & 0.476 & 0.500 & & \\
\hline AA & 0.152 & 0.105 & & \\
\hline \multicolumn{5}{|l|}{ rs1801213 } \\
\hline GG & 0.474 & 0.482 & $1.44(1.10-1.99)$ & 0.007 \\
\hline GC & 0.430 & 0.457 & & \\
\hline $\mathrm{CC}$ & 0.096 & 0.061 & & \\
\hline \multicolumn{5}{|l|}{ rs 734312} \\
\hline AA & 0.295 & 0.309 & $1.22(1.02-1.48)$ & 0.03 \\
\hline AG & 0.489 & 0.527 & & \\
\hline GG & 0.216 & 0.164 & & \\
\hline
\end{tabular}

also observed for the other two SNPs, both at baseline and throughout the study (data not shown).

Cross-sectional study: DIABHYCAR, DIABHYCAR Gene and NCH cohorts Clinical characteristics of participants from the type 2 diabetes cohorts and from the control cohort are shown in ESM Table 4. A logistic regression analysis was performed for each SNP with the cohort membership as the dependent variable, genotype as independent covariable, and sex, age and BMI as adjustment covariables. Odds ratio associated with genotype was computed for each type 2 diabetes cohort against the control cohort (ESM Table 5). Statistically significant associations of the major allele of the three variants with diabetes was confirmed in all type 2 diabetes cohorts individually, as well as with pooled data of the three cohorts. Linkage disequilibrium between the variants was computed and haplotype frequencies were determined for patients and for controls. A logistic regression analysis was performed with glycaemic status as the dependent variable, the haplotypes as independent covariable, and sex, age and BMI as adjustment covariables (ESM Table 6). The GGA haplotype (major allele of the three variants) was significantly associated with diabetes compared with the ACG haplotype (minor alleles of the variants).

\section{Discussion}

We observed statistically significant associations of the major alleles of rs10010131, rs1801213 and rs734312 in the WFS1 locus with an increased risk of type 2 diabetes in individuals with NFG or IFG in a 9 year prospective study in a French cohort issued from the general population. Only a trend towards an association with one of the variants (rs1801213) was observed when we considered the incidence of all hyperglycaemic events (NFG to IFG, NFG to

Table 4 Intermediate phenotypes at baseline according to rs10010131 genotype and glycaemic status at follow-up, the DESIR cohort

\begin{tabular}{|c|c|c|c|c|c|c|c|c|c|}
\hline \multirow{2}{*}{$\begin{array}{l}\text { Glycaemic status } \\
\text { Genotype }\end{array}$} & \multicolumn{3}{|l|}{ NFG } & \multirow[t]{2}{*}{$p$ value } & \multicolumn{3}{|c|}{ IFG/type 2 diabetes } & \multirow[t]{2}{*}{$p$ value } & \multirow{2}{*}{$\begin{array}{l}\text { Interaction } \\
p \text { value }^{\mathrm{a}}\end{array}$} \\
\hline & GG & GA & $\mathrm{AA}$ & & GG & GA & AA & & \\
\hline Participants $(n)$ & 1,111 & 1,421 & 459 & & 400 & 482 & 141 & & \\
\hline FPG (mmol/1) & $5.18 \pm 0.02$ & $5.17 \pm 0.02$ & $5.20 \pm 0.04$ & 0.80 & $6.17 \pm 0.04$ & $6.09 \pm 0.04$ & $5.87 \pm 0.06$ & 0.0005 & 0.002 \\
\hline $\mathrm{HbA}_{1 \mathrm{c}}(\%)$ & $5.40 \pm 0.01$ & $5.39 \pm 0.01$ & $5.41 \pm 0.02$ & 0.64 & $5.82 \pm 0.03$ & $5.71 \pm 0.02$ & $5.61 \pm 0.04$ & $<0.0001$ & $<0.0001$ \\
\hline Fasting insulin $(\mathrm{pmol} / \mathrm{l})$ & $45 \pm 1$ & $44 \pm 1$ & $45 \pm 1$ & 0.47 & $53 \pm 1$ & $57 \pm 1$ & $59 \pm 2$ & 0.31 & 0.60 \\
\hline HOMA\%B (\%) & $77 \pm 1$ & $77 \pm 1$ & $77 \pm 1$ & 0.92 & $65 \pm 1$ & $67 \pm 1$ & $71 \pm 2$ & 0.0002 & 0.0009 \\
\hline HOMA\%S (\%) & $146 \pm 2$ & $147 \pm 1$ & $144 \pm 3$ & 0.86 & $125 \pm 3$ & $124 \pm 3$ & $123 \pm 5$ & 0.23 & 0.34 \\
\hline
\end{tabular}

Unless otherwise stated, data are expressed as mean \pm SEM

Comparisons are analysis of covariance, adjusted for sex, age and BMI, and computed with log-transformed data

a Indicates the statistical significance observed in the regression model for a crossed compound covariable ' $\mathrm{glycaemic}$ status-genotype' 
diabetes or IFG to diabetes change) during follow-up. The variants were also associated with the prevalence of IFG and/or diabetes at baseline. Together, these results suggest that allelic variations of WFS1 increase the risk of diabetes, but have a lesser impact on the risk of IFG in the DESIR cohort. Associations with diabetes were confirmed in crosssectional studies of three cohorts of type 2 diabetic patients compared with normoglycaemic controls. The most frequent haplotype at the haplotype block containing the WFS1 gene was associated with an increased risk of diabetes in the prospective study and with the prevalence of diabetes in cross-sectional studies.

Our results are in agreement with data from the literature. Sandhu and co-workers identified associations of the major alleles of rs10010131, rs6446482, rs752854 and rs734312 SNPs with WFS1 and diabetes risk in 9,533 cases and 11,389 controls from UK and Ashkenazi populations [11]. Lyssenko and co-workers observed an association between the major allele of rs10010131 and an increased risk of diabetes in two prospective cohorts with a median follow-up of 23.5 years and including 16,061 Swedish and 2,770 Finnish participants [13]. An association between rs752854 and type 2 diabetes was also observed in a Swedish cross-sectional study of 1,296 cases and 1,412 controls, with the minor allele conferring protection against diabetes [12]. Other studies have yielded less conclusive results. Florez and co-workers examined the effects of the rs10010131, rs752854 and rs734312 variants on diabetes incidence and response to interventions in the Diabetes Prevention Program, in which a lifestyle intervention or metformin treatment was compared with placebo [28]. They found no statistically significant interactions between genotypes and Diabetes Prevention Program intervention for any of the SNPs. None showed a statistically significant effect on diabetes incidence in the entire cohort, although in the lifestyle arm homozygosis for the minor alleles was consistent with protection from diabetes. Only a trend towards association with diabetes was observed for the rs10010131 variant in a Chinese cross-sectional study of 1,849 type 2 diabetic patients and 1,785 controls [16]. Although it is clear that WFS1 mutations cause diabetes $[7,8]$, the genetic basis of the association of these common WFS1 variants with type 2 diabetes is still unclear. A recent fine-mapping study in UK, Swedish and Ashkenazi populations identified six novel, highly correlated SNPs showing strong and comparable associations with type 2 diabetes risk [29]. None had obvious functional properties that could predict deleterious effects on protein function. Given the strong linkage disequilibrium in the WFS1 region, the authors suggested that further refinement of these associations for identification of the variants responsible for increased diabetes risk would require studies in ethnically diverse populations and/ or studies with much larger sample sizes $(10,000$ to 100,000 individuals) [29].

We also observed statistically significant associations of the risk alleles of the three SNPs with higher FPG and $\mathrm{HbA}_{1 \mathrm{c}}$ levels, and lower HOMA\%B levels, both at baseline and throughout the study in participants who developed hyperglycaemia or type 2 diabetes. Interestingly, no allelic association with these intermediate phenotypes was observed in individuals who remained normoglycaemic at the end of the follow-up. The design of our study had some intrinsic limitations. Glucose tolerance status of participants was based on FPG only and not on an OGTT. Second, insulin secretion and insulin sensitivity were assessed by HOMA indices, which provide only an estimation of these variables. Nonetheless, we do not believe that these limitations have substantially biased our results, as they are in agreement with other observations. Sparso and coworkers observed a similar interaction between the glycaemic status and the genotype effect of rs 734312 on insulin secretion in the Inter99 cohort, which involved 4,568 glucose-tolerant individuals and 1,471 individuals with treatment-naive abnormal glucose regulation (IFG, impaired glucose tolerance or screen-detected diabetes) [14]. The risk allele was associated with a decrease in insulinogenic index and decreased 30 min serum insulin levels after an oral glucose load in individuals with abnormal glucose regulation, but not in participants with normal glucose tolerance. It seems that this interaction between glycaemic status and genotype might explain the heterogeneous results reported by previous studies of associations between WFS1 variants and insulin secretion. Schafer and co-workers studied 1,578 non-diabetic German individuals at increased risk of type 2 diabetes, i.e. persons with a history of gestational diabetes, IFG, impaired glucose tolerance or family history of type 2 diabetes in first-degree relatives [15]. They found that the rs10010131 risk allele was associated with reduced insulin secretion during an OGTT. Interestingly, insulin secretion in response to an IVGTT or a hyperglycaemic clamp was not different between genotypes. However, when the hyperglycaemic clamp was combined with a glucagon-like peptide 1 (GLP-1) infusion, first- and second-phase insulin secretion were significantly decreased in carriers of the risk allele. A study from the Netherlands with similar size and power, but performed in participants with normal glucose tolerance, observed no significant genotype effect on insulin secretion in response to GLP-1 and glucose infusion during a hyperglycaemic clamp [17]. Other studies in large cohorts of non-diabetic Finnish [18] or Chinese [16] participants observed no association between rs10010131 and insulin secretion during an OGTT.

WFS1 protects cells from the damaging effects of hyperactivation of ER stress signalling [3]. ER stress is 
caused by physiological and pathological stimuli that lead to accumulation of a large load of unfolded and misfolded proteins in the ER. For instance, physiological ER stress occurs in pancreatic beta cells during postprandial stimulation of insulin synthesis [30]. Activation of ER stress signalling is tightly regulated because hyperactivation or chronic activation of this signalling pathway can cause cell death by inducing apoptosis [31]. Recent results have shown that WFS1 has an important function in the tight regulation of ER stress through its interaction with activating transcription factor $6 \alpha$ (ATF6 $\alpha$ ), a key transcription factor implicated in ER stress signalling [3]. WFS1 recruits ATF6 $\alpha$ to the proteasome, where in the absence of ER stress it undergoes degradation. In situations of ER stress, ATF6 $\alpha$ is released from WFS1 in the ER membrane and translocates to the nucleus, where it upregulates stress signalling targets. As WFS1 is induced by ER stress, it causes eventual degradation of ATF6 $\alpha$ when ER homeostasis is established.

A possible reason why WFS1 variants only associate with insulin secretion in individuals with abnormal glucose regulation is that these polymorphisms have only a minor impact on WFS1 function in physiological situations. Thus, their effect on insulin secretion can be best appreciated in situations of chronic activation of ER stress signalling, such as in participants with decreased insulin sensitivity and chronic stimulation of insulin synthesis. Moreover, participants with abnormal glucose regulation may have other stressors of beta cell function, including unfavourable alleles in other pancreatic beta cell genes, as well as glucotoxicity, lipotoxicity or defects in the potentiation of insulin secretion by incretins (GLP-1, glucose-dependent insulinotropic polypeptide) [32], which could interact with susceptibility variants of WFS1 and aggravate ER stressmediated apoptosis. WFS1 risk alleles have been shown to be associated with reduced insulin response to oral, but not to intravenous glucose [14, 15], and with reduced response to GLP-1 stimulation during hyperglycaemic clamps [15]. This suggests an incretin-related defect. GLP-1 not only enhances insulin secretion in response to glucose, but also directly modulates the ER stress response, preventing beta cell apoptosis and promoting beta cell adaptation and survival $[32,33]$. At the present time, the mechanisms of the putative interaction of GLP-1 signalling and WFS1 on ER function are still unclear.

In conclusion, allelic variations in the WFS1 gene modulated insulin secretion in normoglycaemic French individuals at risk of developing type 2 diabetes and also increased the risk of type 2 diabetes in a 9 year prospective study. In our study, the most frequent haplotype at the haplotype block containing the WFS1 gene was associated with an increased risk of diabetes. The genetic basis of these associations is still unclear. Further studies with much larger sample sizes are required to identify the functional variants responsible for increased diabetes risk.

Acknowledgements N. Cheurfa was supported by grants from Société Francophone du Diabète (SFD - Alfediam) and Association Diabète Risque Vasculaire (ADRV), France. A. F. Reis was supported by grant 1798-09-0 from CAPES, Brazil. The DESIR study has been financed by: INSERM contracts with CNAMTS, Lilly, Novartis Pharma and Sanofi-Aventis; and by INSERM (Réseaux en Santé Publique, Interactions entre les déterminants de la santé, Cohortes Santé TGIR 2008), the Association Diabète Risque Vasculaire, the Fédération Française de Cardiologie, La Fondation de France, ALFEDIAM, ONIVINS, Ardix Medical, Bayer Diagnostics, Becton Dickinson, Cardionics, Merck Santé, Novo Nordisk, Pierre Fabre, Roche, Topcon. Analysis and interpretation of the data were done without the participation of these organisations.

Duality of interest The authors declare that there is no duality of interest associated with this manuscript.

\section{References}

1. Takeda K, Inoue H, Tanizawa Y et al (2001) WFS1 (Wolfram syndrome 1) gene product: predominant subcellular localization to endoplasmic reticulum in cultured cells and neuronal expression in rat brain. Hum Mol Genet 10:477-484

2. Fonseca SG, Fukuma M, Lipson KL et al (2005) WFS1 is a novel component of the unfolded protein response and maintains homeostasis of the endoplasmic reticulum in pancreatic $\beta$-cells. J Biol Chem 280:39609-39615

3. Fonseca SG, Ishigaki S, Oslowski CM et al (2010) Wolfram syndrome 1 gene negatively regulates ER stress signaling in rodent and human cells. J Clin Invest 120:744-755

4. Lipson KL, Fonseca SG, Ishigaki S et al (2006) Regulation of insulin biosynthesis in pancreatic beta cells by an endoplasmic reticulum-resident protein kinase IRE1. Cell Metab 4:245-254

5. Fonseca SG, Lipson KL, Urano F (2007) Endoplasmic reticulum stress signaling in pancreatic beta-cells. Antioxid Redox Signal 9:2335-2344

6. Riggs AC, Bernal-Mizrachi E, Ohsugi M et al (2005) Mice conditionally lacking the Wolfram gene in pancreatic islet beta cells exhibit diabetes as a result of enhanced endoplasmic reticulum stress and apoptosis. Diabetologia 48:2313-2321

7. Inoue H, Tanizawa Y, Wasson J et al (1998) A gene encoding a transmembrane protein is mutated in patients with diabetes mellitus and optic atrophy (Wolfram syndrome). Nat Genet 20:143-148

8. Strom TM, Hortnagel K, Hofmann S et al (1998) Diabetes insipidus, diabetes mellitus, optic atrophy and deafness (DIDMOAD) caused by mutations in a novel gene (wolframin) coding for a predicted transmembrane protein. Hum Mol Genet 7:2021-2028

9. Barrett TG, Bundey SE, Macleod AF (1995) Neurodegeneration and diabetes: UK nationwide study of Wolfram (DIDMOAD) syndrome. Lancet 346:1458-1463

10. Fraser FC, Gunn T (1977) Diabetes mellitus, diabetes insipidus, and optic atrophy. An autosomal recessive syndrome? J Med Genet 14:190-193

11. Sandhu MS, Weedon MN, Fawcett KA et al (2007) Common variants in WFS1 confer risk of type 2 diabetes. Nat Genet 39:951-953

12. Franks PW, Rolandsson O, Debenham SL et al (2008) Replication of the association between variants in WFS1 and risk of type 2 diabetes in European populations. Diabetologia 51:458-463 
13. Lyssenko V, Jonsson A, Almgren P et al (2008) Clinical risk factors, DNA variants, and the development of type 2 diabetes. N Engl J Med 359:2220-2232

14. Sparso T, Andersen G, Albrechtsen A et al (2008) Impact of polymorphisms in WFS1 on prediabetic phenotypes in a population-based sample of middle-aged people with normal and abnormal glucose regulation. Diabetologia 51:1646-1652

15. Schafer SA, Mussig K, Staiger H et al (2009) A common genetic variant in WFS1 determines impaired glucagon-like peptide-1induced insulin secretion. Diabetologia 52:1075-1082

16. Hu C, Zhang R, Wang C et al (2009) PPARG, KCNJ11, CDKAL1，CDKN2A-CDKN2B，IDE-KIF11-HHEX，IGF2BP2 and SLC30A8 are associated with type 2 diabetes in a Chinese population. PLoS ONE 4:e7643

17. Simonis-Bik AM, Nijpels G, van Haeften TW et al (2009) Gene variants in the novel type 2 diabetes loci CDC123/CAMK1D, THADA, ADAMTS9, BCL11A, and MTNR1B affect different aspects of pancreatic beta-cell function. Diabetes 59:293-301

18. Stancakova A, Kuulasmaa T, Paananen J et al (2009) Association of 18 confirmed susceptibility loci for type 2 diabetes with indices of insulin release, proinsulin conversion, and insulin sensitivity in 5,327 nondiabetic Finnish men. Diabetes 58:2129-2136

19. The D.E.S.I.R. Study Group (1996) An epidemiologic survey from a network of French Health Examination Centres. D.E.S.I.R. (Epidemiologic Data on the Insulin Resistance Syndrome). Rev Epidem Sante Publ 44:373-375

20. Balkau B, Lange C, Fezeu L et al (2008) Predicting diabetes: clinical, biological, and genetic approaches: data from the Epidemiological Study on the Insulin Resistance Syndrome (DESIR). Diab Care 31:2056-2061

21. Lievre M, Marre M, Chatellier G et al (2000) The non-insulindependent diabetes, hypertension, microalbuminuria or proteinuria, cardiovascular events, and ramipril (DIABHYCAR) study: design, organization, and patient recruitment. DIABHYCAR Study Group. Control Clin Trials 21:383-396

22. Marre M, Lievre M, Chatellier G, Mann JF, Passa P, Menard J (2004) Effects of low dose ramipril on cardiovascular and renal outcomes in patients with type 2 diabetes and raised excretion of urinary albumin: randomised, double blind, placebo controlled trial (the DIABHYCAR study). BMJ 328:495

23. Reis AF, Dubois-Laforgue D, Bellanné-Chantelot C, Timsit J, Velho G (2004) A polymorphism in the promoter of UCP2 gene modulates lipid levels in patients with type 2 diabetes. Mol Genet Metab 82:339-344

24. Dina C, Meyre D, Gallina S et al (2007) Variation in FTO contributes to childhood obesity and severe adult obesity. Nat Genet 39:724-726

25. Sladek R, Rocheleau G, Rung J et al (2007) A genome-wide association study identifies novel risk loci for type 2 diabetes. Nature 445:881-885

26. Wallace TM, Levy JC, Matthews DR (2004) Use and abuse of HOMA modeling. Diab Care 27:1487-1495

27. Tregouet DA, Escolano S, Tiret L, Mallet A, Golmard JL (2004) A new algorithm for haplotype-based association analysis: the Stochastic-EM algorithm. Ann Hum Genet 68:165-177

28. Florez JC, Jablonski KA, McAteer J et al (2008) Testing of diabetes-associated WFS1 polymorphisms in the Diabetes Prevention Program. Diabetologia 51:451-457

29. Fawcett KA, Wheeler E, Morris AP et al (2010) Detailed investigation of the role of common and low-frequency WFS1 variants in type 2 diabetes risk. Diabetes 59:741-746

30. Eizirik DL, Cardozo AK, Cnop M (2008) The role for endoplasmic reticulum stress in diabetes mellitus. Endocr Rev 29:42-61

31. Cnop M, Ladriere L, Hekerman P et al (2007) Selective inhibition of eukaryotic translation initiation factor 2 alpha dephosphorylation potentiates fatty acid-induced endoplasmic reticulum stress and causes pancreatic beta-cell dysfunction and apoptosis. J Biol Chem 282:3989-3997

32. Holst JJ, Vilsboll T, Deacon CF (2009) The incretin system and its role in type 2 diabetes mellitus. Mol Cell Endocrinol 297:127-136

33. Yusta B, Baggio LL, Estall JL et al (2006) GLP-1 receptor activation improves beta cell function and survival following induction of endoplasmic reticulum stress. Cell Metab 4:391-406 